01

\title{
Представление потенциала однородного кругового тора рядом по степеням геометрического параметра
}

\section{() Б.П. Кондратьев}

Государственный астрономический институт им. П.К. Штернберга, Московский государственный университет им. М.В. Ломоносова, 119239 Москва, Россия

Главная (Пулковская) астрономическая обсерватория РАН, 196140 Санкт-Петербург, Россия

e-mail: work@boris-kondratyev.ru

(Поступило в Редакцию 17 января 2017 г.)

Поставлена и решена задача о разложении пространственного потенциала однородного гравитирующего (или заряженного статическим электрическим зарядом) кругового тора в ряд по степеням геометрического параметра тора $q$. Первый член этого ряда при $q$ в нулевой степени совпадает с потенциалом тонкого кругового кольца, имеющего массу исходного тора и радиус его осевой линии. Установлено, что все коэффициенты ряда с нечетной степенью геометрического параметра $q$ обращаются в нуль. Четные члены разложения потенциала тора получены в аналитическом конечном виде и выражены через стандартные полные эллиптические интегралы. Важно, что данный ряд представляет потенциал тора во всем пространстве, включая и точки его внутренней области. Данный метод представления потенциала позволил найти гравитационную энергию однородного кругового тора. В качестве приложений впервые была найдена масса каждого из двух колец уникального астероида Chariklo. Масса внутреннего кольца найдена равной $M_{r 1} \approx 9.8 \cdot 10^{18} \mathrm{~g}$, а ее отношение к массе астероида $\frac{M_{r 1}}{M_{0}} \approx 0.001$; аналогично для внешнего кольца астероида получено $M_{r 2} \approx 10^{18} \mathrm{~g}$ и $\frac{M_{r 1}}{M_{0}} \approx 10^{-4}$.

DOI: $10.21883 /$ JTF.2018.03.45584.2169

\section{Введение}

Во многих задачах астрономии, физики и гидродинамики важно знать притяжение (или потенциал) однородного гравитирующего (или заряженного электрическим зарядом) кругового тора с поверхностью

$$
\left(r-R_{0}\right)^{2}+x_{3}^{2}=r_{0}^{2}, \quad r=\sqrt{x_{1}^{2}+x_{2}^{2}} .
$$

Здесь используется система цилиндрических координат $\left(r, x_{3}\right)$ с началом в центре симметрии тора, $R_{0}$ и $r_{0}$ есть радиусы осевой линии и рукава тора соответственно. Впервые пространственный потенциал тора был найден в интегральном виде через суммирование вкладов от однородных круговых дисков [1]. Данный подход позволил получить потенциал тора через однократный интеграл от полных эллиптических интегралов:

$$
\begin{aligned}
& \frac{\varphi_{\mathrm{tor}}\left(r, x_{3}\right)}{2 \sqrt{2} G \rho R_{0} r_{0}}=\int_{0}^{2 \pi}\left\{\left[c+2\left(R_{1}^{2}-\frac{r^{2}}{R_{0}^{2}}\right)\right] K\left(k_{1}\right)\right. \\
& \left.+(a-c) E\left(k_{1}\right)-2 \frac{\left(x_{3}-r_{0} \sin \theta\right)^{2}}{R_{0}^{2}} \Pi\left[n, k_{1}\right]\right\} \\
& \quad \times \frac{\cos \theta d \theta}{\sqrt{a-c}} .
\end{aligned}
$$

Здесь

$$
\begin{gathered}
R_{1}=1+\frac{r_{0}}{R_{0}} \cos \theta, \quad a=\frac{2\left(r^{2}+\left(x_{3}-r_{0} \sin \theta\right)^{2}\right)}{R_{0}^{2}}, \\
n=\frac{a-b}{2 \frac{r^{2}}{R_{0}^{2}}}, \\
\left(\begin{array}{l}
b \\
c
\end{array}\right)=\frac{a}{2}-R_{1}^{2} \pm \sqrt{\left(\frac{a}{2}-R_{1}^{2}\right)^{2}+4 R_{1}^{2} \frac{\left(x_{3}-r_{0} \sin \theta\right)^{2}}{R_{0}^{2}}}
\end{gathered}
$$

а модуль всех эллиптических интегралов

$$
\begin{gathered}
k_{1}=\frac{1-k^{\prime}}{1+k^{\prime}} \leq 1, \\
k^{\prime}=\sqrt{\frac{\left(r-R_{1}\right)^{2}+\left(x_{3}-r_{0} \sin \theta\right)^{2}}{\left(r+R_{1}\right)^{2}+\left(x_{3}-r_{0} \sin \theta\right)^{2}}} \leq 1 .
\end{gathered}
$$

Формула (2) представляет потенциал тора во всем пространстве как вне, так и внутри его.

Заметим, однако, что нахождение потенциала тора в интегральной форме указанным методом через диски не является единственно возможным. Его потенциал можно также найти, например, методом интегрирования вкладов от тонких оболочек кругового тора (см. также [1]). Именно второй метод и применяется в настоящей работе. 
Важно подчеркнуть, что наряду с полным выражением потенциала, часто возникает необходимость знать потенциал какого-то конкретного тела в виде рядов [2]. Отметим, что представления „внешнего “ и „внутреннего“ потенциалов тора в ряд по степеням координат пробной точки (в ряд Лапласа) с коэффициентами в конечном аналитическом виде недавно были получены в работах [3,4]. Кроме того, в работе [5] был развит метод решения задачи Дирихле для потенциалов тел с топологией тора, когда граничные условия заданы в виде рядов по сферическим функциям на кусках двух сферических поверхностей. Этот метод впервые позволил представить потенциал тора вне вещества в особой („промежуточной“) сферической зоне.

В настоящей работе ставится новая задача о представлении пространственного потенциала тора в виде ряда по степеням геометрического параметра тора

$$
q=\frac{r_{0}}{R_{0}}
$$

Этот геометрический параметр может изменяться в пределах

$$
0 \leq q \leq 1
$$

При $q \rightarrow 0$ тор вырождается (с сохранением при определенных условиях массы) в тонкий круглый обруч, а в пределе $q \rightarrow 1$ получается тор без сквозного отверстия.

Кроме ряда Лапласа, для практических целей важно также представить потенциал тора рядом по степеням его геометрического параметра. Этот ряд имеет вид

$$
\varphi_{\text {tor }}\left(r, x_{3}\right)=\varphi_{0}+\sum_{n} \varphi_{n} q^{n} .
$$

Наша задача заключается в нахождении коэффициентов $\varphi_{0}$ и $\varphi_{n}$.

\section{Постановка и решение задачи}

Для решения поставленной задачи мы применим формулу из монографии [1] для пространственного потенциала пустотелого тора

$$
\begin{aligned}
\varphi_{\text {hollow tor }}\left(r, x_{3}\right)= & 4 G \sigma r_{0} \\
& \times \int_{0}^{2 \pi} \frac{R_{1}(\theta) K(k(\theta)) d \theta}{\sqrt{\left[R_{1}(\theta)+r\right]^{2}+\left(x_{3}-r_{0} \sin \theta\right)^{2}}},
\end{aligned}
$$

где введены следующие обозначения:

$$
\begin{gathered}
R_{1}(\theta)=R_{0}+r_{0} \cos \theta, \\
k(\theta)=\sqrt{\frac{4 r R_{1}(\theta)}{\left[R_{1}(\theta)+r\right]^{2}+\left(x_{3}-r_{0} \sin \theta\right)^{2}}},
\end{gathered}
$$

а $\sigma$ - однородная поверхностная плотность вещества тонкой оболочки. Тогда потенциал сплошного однородного (с объемной плотностью $\rho$ ) кругового тора получим, произведя в $(8)$ замену $\sigma \rightarrow \rho d r_{0}$ и интегрируя затем по параметру $r_{0}$ в интервале $\left(0, r_{0}\right)$ :

$$
\begin{aligned}
\varphi_{\text {tor }}\left(r, x_{3}\right)= & 4 G \rho \int_{0}^{r_{0}} r_{0} d r_{0} \\
& \times \int_{0}^{2 \pi} \frac{R_{1}(\theta) K(k(\theta)) d \theta}{\sqrt{\left[R_{1}(\theta)+r\right]^{2}+\left(x_{3}-r_{0} \sin \theta\right)^{2}}} .
\end{aligned}
$$

Подчеркнем, что в отличие от (2), интегрирование в (10) двойное, однако в силу симметрии тора (так как сам тор естественным образом слагается из подобных друг другу оболочек), пользоваться формулой (10) для разложения потенциала по нормированному $r_{0}$ будет удобнее, нежели исходить прямо из формулы (2).

Первый член разложения (7) получается из предельным переходом

$$
r_{0} \rightarrow 0 ; \quad 2 \pi r_{0} \sigma \rightarrow \mu_{0}=\frac{M}{2 \pi R_{0}} .
$$

В итоге находим

$$
\varphi_{0}=\frac{2 G M}{\pi b} K(k)
$$

где $M$ - масса кольца, равная массе исходного тора. Здесь и ниже для сокращения записи приняты обозначения

$$
\begin{gathered}
b=\sqrt{\left(r+R_{0}\right)^{2}+x_{3}^{2}}, \quad \tilde{b}=\sqrt{\left(r-R_{0}\right)^{2}+x_{3}^{2}}, \\
k=\frac{2 \sqrt{R_{0} r}}{b} .
\end{gathered}
$$

Далее переходим к нахождению членов ряда (7) более высокого порядка. Это весьма трудоемкая операция. Прежде всего, важно подчеркнуть, что в разложении (7) все коэффициенты $\varphi_{2 n+1}$ при нечетных степенях $q^{2 n+1}$ обращаются в нуль. Таким образом, мы приходим в выводу о том, что искомый ряд для потенциала тора содержит только четные коэффициенты и поэтому будет иметь вид

$$
\varphi_{\text {tor }}\left(r, x_{3}\right)=\varphi_{0}+\varphi_{2} q^{2}+\varphi_{4} q^{4}+\varphi_{6} q^{6}+\ldots
$$

Далее после трудоемких преобразований и вычислений мы получаем следующие коэффициенты из (13):

$$
\begin{aligned}
& \varphi_{2}=\frac{\pi G \rho R_{0}^{3}}{16 b \tilde{b}^{2}}\left\{\left[r^{2}-R_{0}^{2}+x_{3}^{2}\right] E(k)-\tilde{b}^{2} K(k)\right\} \\
& \varphi_{4}=\frac{\pi G \rho R_{0}^{3}}{192 b^{3} \tilde{b}^{2}} \times \\
& \left\{\frac{\left[\left(r^{2}-2 R_{0}^{2}\right)\left(r^{2}-R_{0}^{2}\right)^{2}+\left(3 r^{4}+R_{0}^{4}\right) x_{3}^{2}+\left(3 r^{2}+4 R_{0}^{2}\right) x_{3}^{4}+x_{3}^{6}\right]}{\left[\left(r-R_{0}\right)^{2}+x_{3}^{2}\right]}\right. \\
& \left.\times E(k)-\left[\left(r^{2}-R_{0}^{2}\right)^{2}+\left(2 r^{2}+3 R_{0}^{2}\right) x_{3}^{2}+x_{3}^{4}\right] K(k)\right\}
\end{aligned}
$$




$$
\begin{aligned}
& \varphi_{6}=\frac{\pi G \rho R_{0}^{5}}{960 b^{3} \tilde{b}^{4}} \\
& \times\left\{\frac{\left[\left(r^{2}-2 R_{0}^{2}\right)\left(r^{2}-r_{0}^{2}\right)^{2}+\left(3 r^{4}+R_{0}^{4}\right) x_{3}^{2}+\left(3 r^{2}+4 R_{0}^{2}\right) x_{3}^{4}+x_{3}^{6}\right]}{\tilde{b}^{2}}\right. \\
& \left.\times E(k)-\left[\left(r^{2}-R_{0}^{2}\right)^{2}+\left(2 r^{2}+3 R_{0}^{2}\right) x_{3}^{2}+x_{3}^{4}\right] K(k)\right\} .
\end{aligned}
$$

Все найденные коэффициенты были тщательно проверены нами численно.

Важным для практических приложений свойством ряда (13) является то, что при малом значении геометрического параметра $q=\frac{r_{0}}{R_{r}} \approx 1$ вклад членов ряда (13) в потенциал тора быстро убывает.

\section{Гравитационная энергия тора при малых $q$}

По определению гравитационная (потенциальная) энергия однородного тела равна

$$
W=-\frac{1}{2} \rho \iiint_{V} \varphi d V .
$$

Вводя среднее (по объему тела) значение потенциала $\tilde{\varphi} \equiv\langle\varphi\rangle$ запишем выражение (17) в виде

$$
W=-\frac{1}{2} M \tilde{\varphi}
$$

Разработанный математический аппарат мы применяем далее к кольцам уникального астероида Chariklo (Карикло). Для изучения колец этого астероида мы будем моделировать их торами. Известно [6], что система колец астероида Chariklo состоит из плотного внутреннего кольца шириной $D_{1}$ и радиусом $R_{r 1}$, а также внешнего кольца шириной $D_{2}$ и радиусом $R_{r 2}$. Кольца имеют четкие границы и между ними существует щель в $9 \mathrm{~km}$. Геометрические параметры колец Chariklo известны

$$
\begin{aligned}
& R_{r 1} \approx 390.6 \pm 3 \mathrm{~km}, \quad D_{1} \approx 6.5 \mathrm{~km}, \\
& R_{r 2} \approx 404.8 \pm 3.3 \mathrm{~km}, \quad D_{2} \approx 2 \mathrm{~km} .
\end{aligned}
$$

Сразу заметим, что кольца этого астероида очень тонкие, поэтому соответствующий им геометрический параметр весьма мал

$$
q_{r 1} \approx 0.008 ; \quad q_{r 2} \approx 0.0025 .
$$

Для столь малых $q$ достаточно (и в этом мы убедились численно) выписать только два коэффициента ряда (13)

$$
\begin{gathered}
\varphi_{0}=\frac{2 G M}{\pi b} K(k), \\
\varphi_{2}=\frac{\pi G \rho R_{0}}{16 b \tilde{b}^{2}}\left\{\left[r^{2}-R_{0}^{2}+x_{3}^{2}\right] E(k)-\tilde{b}^{2} K(k)\right\} .
\end{gathered}
$$

Масса этих колец остается пока неопределенной. Поэтому важной задачей является нахождение массы колец астероида. Из теоремы вириала следует [7], что массу колец можно выразить через их гравитационную энергию, т.е. через средний потенциал $\tilde{\varphi}$ соответствующего тора

$$
M_{r}=\frac{3 \pi}{4} M_{0} \frac{r^{2}}{R_{r}^{2}} \tilde{\varphi}
$$

Важной особенностью применяемого метода является то, что в формуле (22) искомая масса $M_{r}$ кольца выражается через известную нам массу центрального тела астероида $M_{0}$.

В силу круговой симметрии колец для нахождения значения $\tilde{\varphi}$ надо усреднить потенциал (18) только в плоскости меридионального кругового сечения тора

$$
r^{\prime 2}+x_{3}^{2}=r_{0}^{2}, \quad r^{\prime}=r-R_{0} .
$$

В принятой модели средний радиус кольца мы принимаем за $R_{0}$. Теперь задача сводится к нахождению среднего нормированного потенциала тора

$$
\tilde{\varphi}_{m}=\left\langle\frac{\varphi}{\frac{G M_{r}}{2 \pi R_{r}}}\right\rangle=I_{1}+I_{2} .
$$

где, согласно (21):

$$
\begin{gathered}
I_{1}=\frac{8}{\pi} \int_{0}^{\pi} d \alpha \int_{\varepsilon}^{1} \frac{K(k)}{b} R d R \\
I_{2}=\frac{1}{8 \pi} \int_{0}^{\pi} d \alpha \int_{\varepsilon}^{1} \frac{R d R}{b}\left[\left(1+\frac{2 \cos \alpha}{q R}\right) E(k)-K(k)\right], \\
b=\sqrt{4+q^{2} R^{2}+4 q R \cos \alpha}, \quad \tilde{b}=q R_{r} R \\
k=\frac{2 \sqrt{1+q R \cos \alpha}}{b} .
\end{gathered}
$$

Заметим, что в (25) нижний предел $\varepsilon$ во внутреннем интеграле введен для исключения при расчетах бесконечности (дело в том, что при $\varepsilon=0$ для точки интегрирования в центре кругового сечения тора имеет место $R=0$, и тогда модуль $k=1$; но, как известно, эллиптический интеграл первого рода при $K(1)$ имеет логарифмическую расходимость). При достаточно малом $\varepsilon$ мы избегаем указанной сингулярности и можем получить значения $I_{1}$ и $I_{2}$ с требуемой для расчетов точностью.

Численные расчеты по формулам (25) дали следующие результаты. Для модели первого кольца астероида Chariklo мы нашли:

$$
\begin{aligned}
& \varepsilon=4 \cdot 10^{-6}, \\
& I_{1} \approx 14.737, \\
& I_{2} \approx-0.215, \\
& \varphi_{m}=I_{1}+I_{2} \approx 14.522 .
\end{aligned}
$$


Аналогично для модели второго кольца астероида:

$$
\begin{aligned}
& \varepsilon=2 \cdot 10^{-5} \\
& I_{1} \approx 17.166 \\
& I_{2} \approx-0.253 \\
& \varphi_{m}=I_{1}+I_{2} \approx 16.913 .
\end{aligned}
$$

В итоге масса внутреннего кольца и ее отношение к массе астероида оказались соответственно равными

$$
M_{r 1} \approx 9.8 \cdot 10^{18} \mathrm{~g}, \quad \text { a } \quad \frac{M_{r 1}}{M_{0}} \approx 0.001
$$

аналогично для внешнего кольца

$$
M_{r 2} \approx 10^{18} \mathrm{~g} \quad \text { и } \quad \frac{M_{r 2}}{M_{0}} \approx 10^{-4} .
$$

Отношение масс колец равно 10, что типично для спутников астероидов и карликовых планет.

Подчеркнем, что вычисление среднего потенциала $\tilde{\varphi}$ фактически эквивалентно нахождению гравитационной энергии $W$ однородного кругового тора. Действительно, гравитационная энергия колец будет равна [8]:

$$
\begin{aligned}
& W_{r 1}=-\frac{G M_{r 1}^{2}}{4 \pi R_{r 1}} \tilde{\varphi}_{1}, \\
& W_{r 2}=-\frac{G M_{r 2}^{2}}{4 \pi R_{r 2}} \tilde{\varphi}_{2} .
\end{aligned}
$$

\section{Обсуждение}

В настоящей работе потенциал тора впервые был представлен рядом по степеням его геометрического параметра в любой точке пространства, включая и точки внутри самого тора. Указанный ряд быстро сходится, поэтому в конкретных примерах можно ограничиться небольшим числом его членов. Первый член ряда при $q$ в нулевой степени совпадает с потенциалом однородного кругового кольца (обруча), имеющего массу рассматриваемого тора и радиус, равный его осевой линии. Для практических приложений важно заметить, что это элементарное кольцо в отдаленных от тора точках дает потенциал, очень мало отличающийся от потенциала самого тора.

Установлено, что все коэффициенты ряда (13) с нечетной степенью геометрического параметра $q$ обращаются в нуль. Четные члены разложения потенциала представлены в аналитическом конечном виде через стандартные полные эллиптические интегралы Лежандра.

Разработанный метод позволил найти гравитационную энергию однородного кругового тора. Кроме того, в настоящей работе найдена также масса двух колец астероида-кентавра Chariklo. Macca внутреннего кольца найдена равной $M_{r 1} \approx 9.8 \cdot 10^{18} \mathrm{~g}$, а ее отношение к массе астероида $\frac{M_{r 1}}{M_{0}} \approx 0.001$; аналогично для внешнего кольца $M_{r 2} \approx 10^{18} \mathrm{~g}$ и $\frac{M_{r 2}}{M_{0}} \approx 10^{-4}$.

\section{Список литературы}

[1] Кондратьев Б.П. Теория потенциала. Новые методы и задачи с решениями. М.: Мир, 2007.

[2] Антонов В.А., Тимошкова Е.И., Холшевников К.В. Введение в теорию ньютоновского потенциала. М.: Наука, 1988. $270 \mathrm{c}$.

[3] Кондратьев Б.П., Дубровский А.С., Трубищына Н.Г., Мухаметшина Э.Ш. // ЖТФ. 2009. Т. 79. Вып. 2. С. 17-21.

[4] Кондратьев Б.П., Трубищына Н.Г. // ЖТФ. 2010. Т. 81. Вып. 1. С. 23-26.

[5] Кондратьев Б.П., Дубровский А.С., Трубицына Н.Г. // ЖТФ. 2012. Т. 82. Вып. 12. С. 7-10.

[6] Braga-Ribas F., Sicardy B., Ortiz J.L. et al. // Nature. 2014. Vol. 508. P. 72-75.

[7] Kondratyev B.P. // Astrophys. Space Sci. 2016. Vol. 351. N 5. P. 9.

[8] Kondratyev B.P. // Astrophys. Space Sci. 2016. Vol. 361. N 12. P. 1-8. 